Digital Press Social Sciences and Humanities

Représentation de Joko Widodo et de Prabowo Subianto en tant que candidats présidentiels en 2019 sur ballgazette.com et lesechos.fr

B. Wahyudi Joko Santoso, Isfajar Ardinuguroho and Ahmad Yulianto

Proceeding of Conférence internationale sur le français 2018

Joesana Tjahjani, Merry Andriani, Sajarwa, Wening Udasmoro (eds) 


\title{
Représentation de Joko Widodo et de Prabowo Subianto en tant que candidats présidentiels en 2019 sur ballgazette.com et lesechos.fr
}

\author{
B. Wahyudi Joko Santoso*, Isfajar Ardinuguroho et Ahmad Yulianto \\ Universitas Negeri Semarang, Semarang, Indonesia \\ *e-mail : wahyudifr@mail.unnes.ac.id
}

\section{Résumé}

L'élection présidentielle en Indonésie, qui aura lieu en avril 2019, a nommé Joko Widodo et Prabowo Subianto comme candidats. Avant le 10 aout 2018, les deux candidats ont cherché des meilleurs candidats pour leur vice-président. Ils mènent intensivement des lobbies politiques. Les médias publient souvent les divers événements politiques, soit les médias imprimés et soit les médias en ligne, y compris la Gazette de Bali et Les Echos. Le but de cette recherche est de trouver des représentations de Joko Widodo et de Prabowo Subianto sur les deux médias en ligne et quels messages ont-ils transmis aux lecteurs. Pour recueillir des données, nous avons utilisé la méthode de lecture intensive des discours qui représentent les deux candidats sur les deux médias, publiés du janvier au début d'août 2018 et la technique de copier des textes. En analysant nous avons utilisé le modèle de Theo van Leeuwen, Analyse Critique du Discours et la transitivité de Halliday. L'analyse montre que bali-gazette.com et LesEchos.fr ont représenté plus positivement Joko Widodo que Prabowo Subianto. La ressemblance de leur représentation signifie que les deux médias en ligne auraient une préférence pour Joko Widodo (le président et le candidat présidentiel 2019) que Prabowo Subianto (le candidat présidentiel 2019). Leur préférence aurait un motif économique plutôt qu'idéologique parce que la Gazette de Bali est le journal culturel-tourisme-culinaire et les Échos est le journal économique-financier. Donc, il y a la relation entre le discours et (la politique) économique.

\section{Mots-clés}

Représentation, analyse critique du discours, motif économique

\begin{abstract}
The presidential election in Indonesia, which will take place in April 2019, brings up Joko Widodo and Prabowo Subianto as candidates. Before August 10, 2018, the two candidates have sought best candidates for Vice President. They lead intensive political lobbies. The media often publish various political events, printed as well as online media, including the Gazette of Bali and Les Echos. The purpose of this research is to find representations of Joko Widodo and Prabowo Subianto on both online media and what messages they sent to readers. To collect data, we used the method of intensive reading of the discourses that represent the candidates, published from January up to the beginning of August 2018 with the copy texts technique. By analyzing we used Theo van Leeuwen's Critical Discourse Analysis and Halliday's transitivity. The analysis shows that bali-gazette.com and LesEchos.fr represented more positively Joko Widodo than Prabowo Subianto which means that the two media online would have a preference for Joko Widodo (the incumbent and the 2019 candidate 2019) rather than Prabowo Subianto (2019 candidate). Their preference would be an economic motive rather ideological since the Bali Gazette is a culturaltourism-culinary journal and Les Échos is an economic-financial journal. So, there is the relationship between discourse and (policy) economic.
\end{abstract}

\section{Keywords}

Representation, critical discourse analysis, economic motive 


\section{Introduction}

Les élections présidentielles de 2019 en Indonésie entrent dans une nouvelle phase, après que les deux paires de candidats à la présidence et à la vice-présidence se soient inscrites à la Commission électorale générale (KPU) le vendredi 10 août 2018. Enfin, Joko Widodo a choisi Ma'aruf Amin, Président Général du Conseil Indonésien des Oulémas (MUI) en tant que candidat à la vice-présidence. Pendant ce temps, le candidat à la présidentielle Prabowo Subianto, avec certaines considérations également, a choisi Sandiaga Uno comme candidat à la vice-présidence.

Le 28 février 2018, Les Echos.fr a annoncé la réélection du président Joko Widodo comme candidat à la présidentielle 2019-2024 dont le titre est « En Indonésie, Joko Widodo prépare déjà sa réélection ». Puisque le titre implique une politique, alors on pourrait l'appeler un discours politique. Le discours dit politique est, au sens restreint, une forme de la discursivité par laquelle un locuteur (individuel ou collectif) poursuit l'obtention du pouvoir. Par cette définition on tend à faire du discours politique un discours du pouvoir. Cette façon de le concevoir peut-être expliquée par le fait de son importance dans la lutte pour l'accession au pouvoir. Il est difficile, en effet, d'envisager une lutte politique sans discours politique. Mais d'un point de vue plus large, le discours politique peut être approché tout simplement comme une parole publique sur la chose publique. En ce sens, toute forme d'expression qui prend pour objet le mode de gestion des institutions publiques, les personnalités politiques, les différents pouvoirs de l'État, les questions d'intérêt public dans une société relève du discours politique. C'est donc un discours qui témoigne de la préoccupation de l'homme par rapport à la gestion de la cité (http://www.analyse-dudiscours.com/discours-politique).

Il y a deux problématiques dans cette recherche : (1) comment Joko Widodo et de Prabowo Subianto sont-ils représentés sur les deux médias en ligne : bali-gazette.com et LesEchos.fr ? (2) quels messages sont-ils transmis aux lecteurs par les deux médias en ligne au-dessus dans les perspective politique et économique du modèle de CDA van Leeuwen?

L'essor de l'analyse critique du discours ou en anglais « critical discourse analyse » (CDA) a influencé la plupart des branches et sous-branches des sciences humaines en général et linguistique appliquée en particulier. Le CDA considère la langue comme un moyen puissant par lesquelles les idéologies, identités et cultures spécifiques deviennent dominantes dans une société. Les chercheurs du CDA estiment que le choix des interlocuteurs linguistiques reflète leurs intentions, leur idéologie et leur pensée. C'est un moyen efficace pour polariser la société. Les analystes du discours critique identifient et étudient des domaines spécifiques tels que l'injustice, l'inégalité, le racisme, le danger, la souffrance, les préjugés, etc.

L'analyse critique du discours a émergé de la « linguistique critique » développée à l'Université d'East Anglia dans les années 1970 et les termes sont maintenant souvent interchangeables. La sociolinguistique accordait peu d'attention à la hiérarchie sociale et au pouvoir. Le CDA a été développé pour la première fois par l'école de linguistes de Lancaster, dont Norman Fairclough était la figure la plus importante. Ruth Wodak a également apporté une contribution majeure à ce domaine d'étude.

La langue se connecte au social en étant le domaine principal de l'idéologie et en étant à la fois un site et un enjeu de luttes pour le pouvoir. L'idéologie a été appelée la base des représentations sociales des groupes et, dans les versions psychologiques du CDA développées par Teun A. van Dijk et Ruth Wodak, on suppose qu'elle constitue une interface sociocognitive entre les structures sociales et les structures discursives. La dimension historique des études critiques du discours joue également un rôle important.

Nous avons choisi cette approche d'analyse critique du discours (CDA) car cette approche est relativement nouvelle par rapport à d'autres approches, telles que les approches structurelles, pragmatique, sociolinguistique, etc. Nous avons également d'autres considérations, à savoir que l'approche CDA est plus appropriée pour analyser le discours politique. En outre, nous considérons également le bon moment, à savoir l'élection présidentielle en Indonésie qui se tiendra dans huit mois, en avril 2019.

Selon van Dijk (2008), le CDA est un type de recherche analytique discursive qui étudie principalement la manière dont l'abus de pouvoir social, la domination et l'inégalité sont édictés, reproduits et contrés par les textes et les discours dans le contexte social et politique. Avec de telles recherches dissidentes, les analystes du discours critique prennent une position explicite et veulent donc comprendre, dénoncer et finalement résister aux inégalités sociales.

Le CDA doit être compris à la fois comme une théorie et une méthode (Chouliaraki \& Fairclough 1999, p. 16), en ce sens qu'elle offre non seulement une description et une interprétation des discours (Rogers 2004, p. 2). Avant de commencer à aborder la question de cette approche théorique, il est important de bien comprendre ce que nous entendons par les concepts de critique, de discours et d'analyse, et ces termes ont été interprétés de manières différentes et contestées. 
Dans le CDA, la notion de «critique " s'applique avant tout à l'engagement avec les relations de pouvoir associées à la théorie critique de l'école de Francfort. En cela, il plaide contre une vision réaliste, neutre et rationaliste du monde. Au lieu de cela, le rôle est de dénouer les relations de pouvoir cachées, largement construites à travers le langage, et de démontrer et contester les inégalités sociales renforcées et reproduites.

Le discours est un terme contesté et contestable. Le moyen le plus utile de gérer cette contestation provient peut-être des travaux de James Gee (1990). Gee utilise le terme de discours (avec un petit «d» pour parler de la langue utilisée ou de la manière dont la langue est utilisée dans un contexte social pour «faire connaître» des activités et des identités). C'est ainsi que les linguistes appliqués tels que McCarthy (1994) ont utilisé le terme pour parler de la langue au-delà du niveau de la phrase - une avancée analytique qui nous permet d'examiner certaines des choses qui ne sont observables que si nous regardons au-delà des exemples de phrase unique, par exemple le mot «This» peut être utilisé au début d'une discussion pour mettre en avant le sujet en discussion et l'identifier comme important pour le locuteur, alors que le mot «That» pourrait être utilisé pour créer un contexte ou marginaliser un sujet et placez-le en tant que position subordonnée, du point de vue du locuteur.

Mais Gee note que le langage ne se produit pas de manière isolée, mais dans des contextes sociaux spécifiques. Il se produit entre des personnes, dans des endroits particuliers ; en particulier, des ensembles de circonstances, à des moments particuliers, accompagnés de signes sémiotiques particuliers (tels que des gestes, des vêtements et des symboles) et influencés par un éventail de valeurs, d'attitudes, de croyances, d'émotions et d'idéologies. C'est ce «truc » non linguistique que Gee qualifie de Discours (avec un grand "D »). Donc, le discours se produit dans les Discours. Pour Gee, "les discours sont caractéristiques (socialement et culturellement formés, mais changent historiquement) des manières de parler et d'écrire, ainsi que d'agir avec et envers les gens et les choses. Ces moyens sont diffusés et soutenus dans divers textes, artefacts, images, pratiques sociales et institutions, ainsi que dans des interactions sociales ponctuelles. À leur tour, elles amènent certaines perspectives et certains états à apparaître ou à être considérés comme " normaux » ou " naturels » et d'autres à paraître ou à être considérés comme « déviants » ou " marginaux » (p. Ex. "Prisonnier, patient à l'hôpital ou étudiant, ou prison" normale ", hôpital ou école, à un moment et à un endroit donnés"

Selon Fairclough (1989, p. 19), l'utilisation de ce langage est appelée pratique socioculturelle et cette pratique est déterminée par la structure sociale existante au moment de la production du discours. Ainsi, le discours n'est pas une entité neutre, mais une entité conçue par certains acteurs sociaux qui contrôlent le processus de communication à des fins politico-économiques. Donc, derrière le discours, il y a une "réalité physique" qu'elle représente. Pour plus de détails, voir les déclarations de constructivistes extrêmes, tels que Potter (1997) d'une part et les réalistes critiques, tels que Sperber (1996), Kress et van Leeuwen (2001) du côté suivant dans Schneider en PoliticsEastAsia (2013) :

Discourse theorists disagree on which parts of our world are real. In other words, they take different ontological stances. Extreme constructivists argue that all human knowledge and experience is socially constructed, and that there is no reality beyond discourse (Potter, 1997). Critical realists, on the other hand, argue that there is a physical reality that "talks back" as we engage with it (Sperber 1996), but that this reality is represented through discourse (Kress \& van Leeuwen, 2001).

Selon Porter, toutes les connaissances et expériences humaines sont construites socialement et il n'y a pas de réalité en dehors du discours. Quant à Sperber, la réalité physique est « rediscutée » lorsque nous y sommes impliqués (Sperber 1996). Cependant, cette réalité est ramenée par le discours (Kress et van Leeuwen, 2001). En d'autres termes, à travers le discours, les médias veulent transmettre certains messages (intentions) de manière implicite ou explicite.

Si on associe à l'une des caractéristiques de CDA est l'action, les médias en ligne (les Echos.fr et baligazzette.com) qui publient la candidature de Joko Widodo et Prabowo Subianto en tant que candidats à la présidence de la République d'Indonésie 2019-2024 entendent transmettre certaine action aux lecteurs, comme accorder ou désaccorder l'idée ou la volonté de journaliste. Selon Tebba (2005), les médias ont une préférence pour les candidats à la présidence. En général, ce soutien repose sur certains motifs, généralement idéologiques, politiques et économiques. En d'autres termes, le CDA n'examine pas seulement des aspects internes (formels), mais révèle plutôt des aspects externes, tels que les trois motifs ci-dessus. Ainsi, il existe une relation significative entre le langage et le pouvoir dans des pratiques imaginaires mais réelles, puis la relation se manifeste comme une idéologie d'intérêts (Fairclough, 1989, p.v). 


\section{Méthode}

Pour collecter des données, nous avons utilisé la méthode de lecture intensive (Sudaryanto, 1993) : c'est la lecture des textes (nouvelles) des candidats à la présidence Joko Widodo et Prabowo Subianto à l'élection présidentielle 2019 dans les deux médias de masse en ligne : bali-gazette.com et LesEchos.fr rapportés pendant 8 mois: janvier - août 2018. Sur bali-gazette.com, nous avons trouvé cinq discours concernant les publications de nomination présidentielle de Joko Widodo et Prabowo Subianto en 2019 et pour les exemples d'analyse, nous n'avons qu'analysé trois discours dont les titres sont (i) « La Guerre Electorale En Indonésie A Déjà Commence... En Ligne », (ii) «Prabowo Subianto en Lice Pour la Presidentielle de 2019», et (iii) «Le Président Indonésien Jokowi Seul en Lice Pour 2019». En même temps que pendant 8 mois, nous n'avons que trouvé un discours concernant la même chose sur LesEchos.fr dont le titre est « En Indonésie, Joko Widodo prépare déjà sa réélection ».

Le choix de cette période est basé sur la période de candidature présidentielle jusqu'à l'inscription au (Commission Électorale Générale). En outre, l'utilisation de la méthode a été suivie par l'utilisation de techniques de copier-coller les données de deux médias en ligne ci-dessus. Les données de recherche sont des citations contenant des publications des deux candidats à la présidentielle : Joko Widodo et Prabowo Subianto. La sélection de bali-gazette.com est basée sur la considération qu'il est centré en Indonésie (à Bali) alors que la sélection de « lesEchos.fr » est basée sur l'idée que ces médias basés en France (à Paris). Les méthodes d'analyse des données suivent le modèle de Fairclough (1989) : ce sont la description, l'interprétation, et l'explication. D'abord, les données sont décrites textuellement, et puis les descriptions des textes sont interprétées afin de déterminer la signification basée sur l'explication des contextes historique, idéologique, politique et économique.

\section{Résultat et discussion}

Selon van Leeuwen (2008, p. 28), le terme « représentation » désigne la manière dont une personne, un groupe ou une idée est affichée dans les publications des médias. En ce qui concerne ces représentations, van Leeuwen, dans son cadre, s'appuie sur des inventaires socio-sémantiques. Selon lui, (1) la suppression, (2) le réarrangement ou l'attribution des rôles et (3) la substitution sont les trois principaux types de transformation permettant de disséquer la représentation des acteurs sociaux (Joko Widodo \& Prabowo Subianto). Selon les trois transformations, les acteurs sociaux peuvent être affichés verbalement et / ou non verbalement (visuellement).

Par ailleurs, selon Sujiman et Zoest (1992), les médias prennent trois mesures pour construire la réalité, ce qui conduit à la formation de représentations (images) : (1) la sélection des symboles, (2) la sélection des faits, et (3) la volonté de fournir une place (un réglage) dans les publications aux médias. En ce qui concerne la couverture de l'actualité, à savoir pendant les huit mois de reportages (janvier-août 2018) Selon van Leeuwen (2008, p. 28), le terme « représentation » désigne la manière dont une personne, un groupe ou une idée est affichée dans les publications des médias. En ce qui concerne ces représentations, van Leeuwen, dans son cadre, s'appuie sur des inventaires socio-sémantiques. Selon lui, (1) la suppression, (2) le réarrangement ou l'attribution des rôles et (3) la substitution sont les trois principaux types de transformation permettant de disséquer la représentation des acteurs sociaux (Joko Widodo et Prabowo Subianto). Selon les trois transformations, les acteurs sociaux peuvent être affichés verbalement et / ou non verbalement (visuellement).

\subsection{Suppression}

Van Leeuwen (2008, p. 28) indique que la « suppression » est une préoccupation majeure dans l'analyse critique du discours, qui consiste en des processus d'inclusion et d'exclusion. Cet expert déclare que «les représentations incluent ou excluent les acteurs sociaux d'ajuster leurs intérêts et objectifs par rapport aux lecteurs pour lesquels ils sont inclus dans les informations. L'exclusion est l'un des processus d'élimination ou de suppression des acteurs sociaux de la publication. Van Leeuwen estime qu'un aspect important du CDA est l'exclusion (2008, pp. 28-34). L'exclusion est un phénomène courant dans les médias et les discours politiques. Et puis, l'inclusion est un autre processus de représentation en termes de « suppression ». L'inclusion des acteurs sociaux est utilisée pour répondre aux besoins et aux objectifs des producteurs des discours médiatiques et des lecteurs. Pour plus de détails, voyez la Table 1 ci-dessous 
Tableu 1 La guerre électorale en Indonésie a déjà commencé... en ligne

\begin{tabular}{|l|l|c|c|}
\hline \multicolumn{2}{|c|}{ Inclusion/Exclusion } & Jokowi Widodo & Prabowo Subianto \\
\hline Inclusion & 11 & 2 \\
\hline \multirow{2}{*}{ Exclusion } & Suppression & 0 & 0 \\
\cline { 2 - 4 } & Backgrounding & 22 & 27 \\
\hline
\end{tabular}

À la Table 1 au-dessus, Joko Widodo a été inclus 11 fois parce que d'un côté, d'après nous, il est le président de la République Indonésienne, l'acteur social social-politique le plus élevé et le plus important en Indonésie. Au nom de la loi et du peuple indonésien que lui a donné à l'élection présidentielle de 2014 dernière. En tant que président actuel, il est responsable de la sécurité de toutes les nations et tout territoire indonésien. Donc, il a le grand pouvoir pour contrôler toutes les perturbations sociales et politiques avant les élections législatives et présidentielles de 2019. En revanche, le journaliste de Bali-Gazette n'a qu'inclus Prabowo Subianto 2 fois. Cela veut dire que le journaliste ne voulait pas publier Prabowo Subianto beaucoup de fois ; ce n'était que 2 fois. Selon Sujiman et Zoest (1992), les médias prennent trois mesures pour construire la réalité, ce qui conduit à la formation de représentations des acteurs sociaux : (1) la sélection des symboles, (2) la sélection des faits, et (3) la volonté de fournir une place (un réglage) dans les publications aux médias. Lié à la sélection de symbole non-verbal, la figure de Prabowo Subianto a mois de valeur politique que Joko Widodo qui était représenté 11 fois et c'était pourquoi le journaliste ne voulait pas donner à Prabowo un réglage suffisant dans le contexte social-politique en ce moment-là.

De l'autre côté, Joko Widodo a été exclu d'une façon «backgrounding» 22 fois. C'est-à-dire qu'il a été exclu du discours mais qu'on pourrait encore reconnaitre sa présence dans les publications aux médias par l'intermédiaire d'une personne (Ahok, ...) ou d'un groupe de personnes (des Communistes, des Chinois, ...) ou d'une institution (la police indonésienne, l'armée indonésienne, ...) ou bien des provinces comme Java-Centre, Java-Est, et Java-Ouest. Lui aussi, Prabowo a été exclu de la même façon « backgrounding » mais on pourrait encore reconnaitre sa présence 27 fois par un groupe de personnes (la Muslim Cyber Army, cyber djihadistes, Saracen, ...) ou bien des noms, comme cyber djihadistes, une ligne islamiste et anti-gouvernementale, ....

Les choix et le montant de lexicaux au-dessus se correspondent bien aux contextes social-politique et historiques de l'élection présidentielle en 2014. La plupart des arguments soutiennent les arguments de l'auteur avec des faits et des statistiques. La nouvelle commence avec des articles qui semblent favoriser Jokowi. Il y a deux parties qui s'opposent, à savoir Jokowi d'un côté et un réseau clandestin diffusant des discours de haine et des canulars en ligne. C'est assez inquiétant. Cela se voit quand on dit que le cyberguerre empêchera Jokowi d'être réélu. «Peut-elle éviter la réélection annoncée de Jokowi? » Cependant, l'auteur n'a pas le courage de relier explicitement Prabowo à ce que l'on appelle le musulman intransigeant.

Les autres citations traitent de la cartographie politique de Jokowi et Prabowo basée sur la précédente élection présidentielle de 2014. Jokowi a dominé Prabowo dans le centre de Java tandis que Prabowo a gagné avec une légère marge à Java-Ouest. La véritable bataille se jouera donc à Java-Ouest et à Java-Est. "Car tout s'est joué sur Java, Prabowo a très largement remporté Java-Ouest, la province la plus peuplée du pays, avec près de 5 millions de voix d'avance. A Java-Centre, c'est Jokowi qui mène de près de 6 millions de voix. Java-Est fut bien plus serrée avec Jokowi en avance d'un million de voix. »

Ensuite, dans la Table 2 suivante, on pourrait voir que Joko Widodo a été inclus 4 fois tandis que Prabowo Subianto a été inclus 3 fois dans le discours dont le titre est « Prabowo Subianto en Lice Pour la Présidentielle de 2019 ». Cela signifie qu'ils ont été exclus dans le même montant : 10 fois. Politiquement et sociologiquement, cela signifie que le journaliste de Bali-Gazette a sélectionné (i) le même symbole : les deux candidats du président indonésien en 2019, (2) le même fait : l'élection présidentielle en 2019, et (3) la même volonté de fournir le réglage ballant dans la publication à bali.gazette.com.

Qui va gagner le concours de l'élection présidentielle de l'année prochaine ? Cela dépend de nombreux facteurs, y compris les machines des partis politiques, la logistique, les volontaires et l'intelligence des électeurs, surtout les électeurs débutants-les jeunes milléniales.

Tableu 2 Prabowo Subianto en lice pour la présidentielle de 2019 Bali.gazette.com

\begin{tabular}{|c|c|c|c|}
\hline \multicolumn{2}{|c|}{ Inclusion/Exclusion } & Joko Widodo & Prabowo Subianto \\
\hline \multicolumn{2}{|c|}{ Inclusion } & 4 & 3 \\
\hline \multirow{2}{*}{ Exclusion } & Suppression & 0 & 0 \\
\cline { 2 - 4 } & Backgrounding & 10 & 10 \\
\hline
\end{tabular}


Et puis, dans la Table 3 suivante, on pourrait voir que Joko Widodo a été inclus 5 fois tandis que Prabowo Subianto a été inclus 2 fois dans le discours dont le titre est «Le Président Indonésien Jokowi Seul en Lice Pour 2019». D’après nous, cela signifie que le journaliste favorise mieux Joko Widodo que Prabowo Subianto parce qu'il leur a donné le réglage tout à fait diffèrent $(5: 2)$. En outre, ils ont également été exclus du discours avec un ratio de $9: 23$ (9 pour Joko Widodo et 23 pour PrabawaSubianto) d'une manière «backgrounding» comme son rival (1), cinq des dix partis au parlement (1), Golkar (1), PDI-P (1), chef du principal parti d'opposition Gerindra (1), son parti (1), son secrétaire général Eddy Soeparno (1), il (3), une coalition (2), Le Parti de la justice prospère (1), le Parti du mandat national (1), plusieurs candidats (1), etc.

Tableu 3 Le président indonésien Jokowi seul en lice pour 2019Bali.gazette.com

\begin{tabular}{|c|c|c|c|}
\hline \multicolumn{2}{|c|}{ Inclusion/Exclusion } & Joko Widodo & Prabowo Subianto \\
\hline \multicolumn{2}{|c|}{ Inclusion } & 5 & 2 \\
\hline \multirow{2}{*}{ Exclusion } & Suppression & 0 & 0 \\
\cline { 2 - 4 } & Backgrounding & 9 & 23 \\
\hline
\end{tabular}

Enfin, dans la Table 4 suivante, on pourrait voir que Joko Widodo a été inclus 8 fois tandis que Prabowo Subianto a été inclus 5 fois dans le discours dont le titre est «En Indonésie, Joko Widodo prépare déjà sa réélection». A notre avis, cela signifie que le journaliste Les Echos.fr préfère Joko Widodo que Prabowo Subianto parce qu'il leur a donné le réglage avec le ratio de $8: 5$ ). De leur côté, ils ont aussi également été exclus du discours avec un ratio de $7: 11$ (7 pour Joko Widodo et 11 (pour PrabawaSubianto) d'une manière «backgrounding» comme le chef de l'Etat (1), tout candidat (1), les deux prétendants au fauteuil suprême (1), le duo (1), le co-fondateur de Gerindra (1), le principal parti d'opposition (1), le candidat malheureux de 2014 (1), son challenger malheureux 2014 1), son concurrent (1), l'opposant (1), son adversaire (1), les deux hommes (1), etc.

Tableu 4 En Indonésie, Joko Widodo prépare déjà sa réélectionLesEchos.fr

\begin{tabular}{|c|c|c|c|}
\hline \multicolumn{2}{|c|}{ Inclusion/Exclusion } & Joko Widodo & Prabowo Subianto \\
\hline \multicolumn{2}{|l|}{ Inclusion } & 8 & 5 \\
\hline \multirow[t]{2}{*}{ Exclusion } & Suppression & 0 & 0 \\
\hline & Backgrounding & 7 & 11 \\
\hline
\end{tabular}

\subsection{Allocation de rôle (réarrangement)}

Ensuite, le « réarrangement » tente d'identifier quels rôles attribués à certains acteurs sociaux par le biais de caractéristiques grammaticales, telles que l'activation, la passivation, la bénéficiassions (van Leeuwen, 2008). Selon Halliday (1985), cela correspond à la transitivité. Pour bien la comprendre, voyez la citation suivante :

Traditionally, transitivity is normally understood as the grammatical feature, which indicates if a verb takes a direct object; and we know some of the terms below:

a. If the verb takes a direct object, then it is described as transitive, and

b. It is called intransitive if it does not;

c. An extension of this concept is the ditransitive verb, which takes both a direct and an indirect object.

Halliday, however, found the new concept of transitivity. The new concept represents a further development of the old concept. In Halliday's conception in his Introduction to Functional Grammar, whether a verb takes or does not take a direct object is not a prime consideration. There are three components of what Halliday calls a "transitivity process", namely:

a. The process itself, 
b. Participants in the process; and

c. Circumstances associated with the process

Then, Halliday divides the system of transitivity or process types into six processes, namely: material, mental, relational, behavioral, verbal, and existential. In connection with the transitivity, let us see the definition of clause: "A clause in English is the simultaneous realization of ideational, interpersonal and textual meanings" (Halliday, 1981, p. 42) "A clause is the product of three simultaneous semantic processes. It is at one and the same time a representation of experience (ideational), an interactive exchange (interpersonal), and a message (textual) (Halliday, 1985, p. 53). From the quotations above, we can conclude that a clause has a close relation to the ideational (both function and the meaning), so that absolutely a clause also can be related to the transitivity. The relation in here is we can analyze a clause by the transitivity system or what Halliday calls as analyzing the meaning of clause as representation.

Basée sur la citation de Halliday au-dessus, la transitivité se divise en 3 groupe : ce sont (a) le processus lui-même, (b) les participants dans le processus, et (c) les circonstances associées au processus. Pour analyser l'allocation de rôle, nous nous basons sur ces concepts. D'après le tableu 5,6 , et 7, ci-dessous, Joko Widodo était représenté par le journaliste comme « agent » 17 fois, comme «patient » 12 fois, et comme « récipient » 5 fois. Par contre, Prabowo Subianto était représenté comme « agent » 10 fois, comme « patient » 24 fois, et comme « récipient » 2 fois.

D'abord, basée de ces données, cela signifie que comme « agent » Joko Widodo était représenté comme un acteur social (une figure) plus actif, plus puissant, plus important, et plus super que Prabowo Subianto. Ensuite, comme " patient » Prabowo Subianto était représenté comme un acteur social étant moins actif, moins puissant, moins important, et moins super que Joko Widodo. Enfin, comme " récipient » Joko Widodo était représenté comme un acteur social était plus bénéficié que Prabowo Subianto. Politiquement, toutes les différences de cette signification donnent un impact politique aux lecteurs soit directement ou soit indirectement

Tableu 5 La guerre électorale en Indonésie a déjà commencé... en ligne Bali.gazette.com

\begin{tabular}{|c|c|c|}
\hline Activated/Subjected/Beneficialized & Joko Widodo & Prabowo Subianto \\
\hline Activated ("actor" /"agent") \% & 12 & 4 \\
\hline Subjected ("patient" /"goal") \% & 6 & 15 \\
\hline Beneficialized ("récipient') \% & 2 & 0 \\
\hline
\end{tabular}

Tableu 6 Prabowo Subianto en lice pour la presidentielle de 2019 Bali.gazette.com

\begin{tabular}{|c|c|c|}
\hline Activated/Subjected/Beneficialized & Joko Widodo & Prabowo Subianto \\
\hline Activated ("actor" /"agent") \% & 1 & 4 \\
\hline Subjected ("patient"/"goal") \% & 5 & 5 \\
\hline Beneficialized ("récipient') \% & 2 & 1 \\
\hline
\end{tabular}

Tableu 7 Le Président indonésien Jokowi seul en lice pour 2019 Bali.gazette.com

\begin{tabular}{|c|c|c|}
\hline Activated/Subjected/Beneficialized & Joko Widodo & Prabowo Subianto \\
\hline Activated ("actor" /"agent") \% & 4 & 2 \\
\hline Subjected ("patient" /"goal") \% & 1 & 4 \\
\hline Beneficialized ("récipient') \% & 1 & 1 \\
\hline
\end{tabular}

D’après la Table 8 ci-dessous, le journaliste de « LesEchos.fr » avait aussi représenté Joko Widodo comme un acteur social (agent) plus actif, plus puissant, plus important, et plus super que Prabowo Subianto. Mais apparemment, comme «patient » Joko Widodo était représenté comme un acteur social étant moins actif, moins puissant, moins important, et moins super que Prabowo Subianto. Si nous lisons le texte apparenté, en fait, le journaliste avait représenté la croissance économique n'ayant pas atteint $7 \%$, le succès de Jokowi sur les 225 projets d'infrastructures prioritaires et sa popularité d'après quelques 
sondages réputés. Et aussi il a déjà une exigence en tant que candidat à la présidence, à savoir au moins $20 \%$ des sièges au Parlement et au moins $25 \%$ des votes aux dernières élections législatives. Enfin, comme " récipient » Joko Widodo était beaucoup plus bénéficié que Prabowo Subianto. Politiquement, toutes les différences de cette signification donnent un impact politique aux lecteurs soit directement ou soit indirectement.

Tableu 8 En Indonésie, Joko Widodo prépare déjà sa réélection LesEchos.fr

\begin{tabular}{|c|c|c|}
\hline Activated/Subjected/Beneficialized & Joko Widodo & Prabowo Subianto \\
\hline Activated ("actor" /"agent") \% & 17 & 13 \\
\hline Subjected ("patient" /"goal") \% & 9 & 0 \\
\hline Beneficialized ("récipient') \% & 10 & 0 \\
\hline
\end{tabular}

\subsection{Substitution}

La « substitution » peut être faite avec plusieurs caractéristiques discursives et à travers ces caractéristiques, les acteurs sociaux sont représentés. Van Leeuwen (2008) déclare qu'il existe plusieurs caractéristiques discursives pour représenter les acteurs sociaux à travers deux substitutions, à savoir : la personnalisation et l'impersonnalisation. La personnalisation est les acteurs sociaux qui sont représentés individuellement/ personnellement. La personnalisation est divisée en 4 sous-substitutions, à savoir la détermination, l'indétermination, la généralisation, et la spécification. L'impersonnalisation est divisée en 2 sous-substitutions, à savoir l'abstraction et l'objectivation. L'impersonnalisation se produit lorsque les acteurs sociaux sont représentés par des noms concrets ou par des caractéristiques sémantiques. La fonctionnalisation sont les acteurs sociaux étant représentés par les rôles ou fonctions qui leur sont attachés et les nominations sont les acteurs sociaux son étant représentés conformément à leurs noms, par exemple de manière informelle, semi-informelle et formelle.

Basées sur les Tables 9-12, d'un côté, les deux journalistes (bali.gazette.com et LesEchos.fr) ont représenté les deux candidats à la présidentielle avec la plupart de la même substitution discursive « personnalisation » de sous-type détermination-catégorisation-fonctionnalisation-identification mais avec des montants différents. Ceux de Jokowi a la tendance plus nombreuse que ceux de Prabowo. Et de l'côté, ils les ont représentés avec une autre substitution discursive " personnalisation » de sous-type nomination-formalisation et informatisation. Mais, il y a une différence entre les deux candidats : les deux journalistes ont utilisé aussi une autre substitution du type personnalisation et du sous-type détermination-association pour représenter Prabowo, à savoir qu'il était représenté comme un candidat ayant la relation sociale de droite extrême (l'Islam radical) et le candidat malheureux de 2014 et aussi le challenger malheureux en 2014 de Jokowi. Il a aussi été représenté " autoritaire » et il a divorcé de sa femme en 1998. Politiquement, toutes les différences d'utilisation de la substitution donnent un impact politique très sérieux aux lecteurs soit directement ou soit indirectement. Pour rendre clair voyons la Table 9-12 ci-dessous.

Tableu 9 La guerre électorale en Indonésie a déjà commencé... en ligne Bali.gazette.com

\begin{tabular}{|c|c|c|c|c|c|}
\hline & Personalizatio & Impersonaliza & & Les D & Candidats \\
\hline & & & & & \\
\hline Personalization & Determination & Categorization & Functionalization & 3 & 1 \\
\hline & & & $\begin{array}{l}\text { Identification } \\
\text { (relational } \\
\text { indentification) }\end{array}$ & $12+3$ & $4+3$ \\
\hline & & & Appraisement & 0 & 2 \\
\hline & & Nomination & Formalization & 0 & 0 \\
\hline & & & $\begin{array}{l}\text { Semi } \\
\text { Formalization }\end{array}$ & 4 & 2 \\
\hline & & & Informalization & 4 & 2 \\
\hline & & Association & & 0 & 2 \\
\hline & & $\begin{array}{l}\text { Single } \\
\text { Determination }\end{array}$ & & 0 & 0 \\
\hline
\end{tabular}




\begin{tabular}{|l|l|l|l|}
\hline \multirow{4}{*}{ Impersonalization } & Indetermination & 0 & 0 \\
\cline { 2 - 4 } & Genericization & 0 & 0 \\
\cline { 2 - 4 } & Specification (Individualization) & 0 & 0 \\
\cline { 2 - 4 } & Abstraction & 0 & 0 \\
\cline { 2 - 4 } & Objectivation & 0 & 0 \\
\hline
\end{tabular}

Tableu 10 Prabowo Subianto en lice pour la présidentielle de 2019Bali.gazette.com

\begin{tabular}{|c|c|c|c|c|c|}
\hline \multicolumn{4}{|c|}{ Personalization/Impersonalization } & \multicolumn{2}{|c|}{ Les Deux Candidats } \\
\hline \multirow[t]{11}{*}{ Personalization } & \multirow[t]{8}{*}{ Determination } & \multirow[t]{3}{*}{ Categorization } & Functionalization & \begin{tabular}{|l|} 
Joko \\
Widodo \\
3
\end{tabular} & $\begin{array}{l}\text { Prabowo } \\
\text { Subianto } \\
1\end{array}$ \\
\hline & & & $\begin{array}{l}\text { Identification } \\
\text { (relational } \\
\text { indentification) }\end{array}$ & $12+3$ & $4+3$ \\
\hline & & & Appraisement & 0 & 2 \\
\hline & & \multirow[t]{3}{*}{ Nomination } & Formalization & 0 & 0 \\
\hline & & & $\begin{array}{l}\text { Semi } \\
\text { Formalization }\end{array}$ & 4 & 2 \\
\hline & & & Informalization & 4 & 2 \\
\hline & & Association & & 0 & 2 \\
\hline & & $\begin{array}{l}\text { Single } \\
\text { Determination }\end{array}$ & & 0 & 0 \\
\hline & \multicolumn{3}{|l|}{ Indetermination } & 0 & 0 \\
\hline & \multicolumn{3}{|c|}{ Genericization } & 0 & 0 \\
\hline & \multicolumn{3}{|c|}{ Specification (Individualization) } & 0 & 0 \\
\hline \multirow[t]{2}{*}{ Impersonalization } & \multicolumn{3}{|c|}{ Abstraction } & 0 & 0 \\
\hline & \multicolumn{3}{|l|}{ Objectivation } & 0 & 0 \\
\hline
\end{tabular}

Tableu 11 Le Président Indonésien Jokowi Seul en Lice Pour 2019 Bali.gazette.com

\begin{tabular}{|c|c|c|c|c|c|}
\hline & Personalizatiol & mpersonalizat & & Les De & Candidats \\
\hline & & & & & \\
\hline Personalization & Determination & Categorization & Functionalization & 3 & 1 \\
\hline & & & $\begin{array}{l}\text { Identification } \\
\text { (relational } \\
\text { indentification) }\end{array}$ & $12+3$ & $4+3$ \\
\hline & & & Appraisement & 0 & 2 \\
\hline & & Nomination & Formalization & 0 & 0 \\
\hline & & & $\begin{array}{l}\text { Semi } \\
\text { Formalization }\end{array}$ & 4 & 2 \\
\hline & & & Informalization & 4 & 2 \\
\hline & & Association & & 0 & 2 \\
\hline & & $\begin{array}{l}\text { Single } \\
\text { Determination }\end{array}$ & & 0 & 0 \\
\hline & Indeterminatio & & & 0 & 0 \\
\hline & Genericization & & & 0 & 0 \\
\hline & Specification (I & ividualization) & & 0 & 0 \\
\hline
\end{tabular}




\begin{tabular}{|l|l|l|l|}
\hline \multirow{2}{*}{ Impersonalization } & Abstraction & 0 & 0 \\
\cline { 2 - 4 } & Objectivation & 0 & 0 \\
\hline
\end{tabular}

Tableu 12 En Indonésie, Joko Widodo prépare déjà sa réélection LesEchos.fr

\begin{tabular}{|c|c|c|c|c|c|}
\hline \multirow{2}{*}{\multicolumn{4}{|c|}{ Personalization/Impersonalization }} & \multirow{2}{*}{\multicolumn{2}{|c|}{ Les Deux Candidats }} \\
\hline & & & & & \\
\hline \multirow[t]{11}{*}{ Personalization } & \multirow[t]{8}{*}{ Determination } & \multirow[t]{3}{*}{ Categorization } & Functionalization & $\begin{array}{l}\text { Joko } \\
\text { Widodo } \\
3\end{array}$ & $\begin{array}{l}\text { Prabowo } \\
\text { Subianto } \\
1\end{array}$ \\
\hline & & & $\begin{array}{l}\text { Identification } \\
\text { (relational } \\
\text { indentification) }\end{array}$ & $12+3$ & $4+3$ \\
\hline & & & Appraisement & 0 & 2 \\
\hline & & \multirow[t]{3}{*}{ Nomination } & Formalization & 0 & 0 \\
\hline & & & $\begin{array}{l}\text { Semi } \\
\text { Formalization }\end{array}$ & 4 & 2 \\
\hline & & & Informalization & 4 & 2 \\
\hline & & Association & & 0 & 2 \\
\hline & & $\begin{array}{l}\text { Single } \\
\text { Determination }\end{array}$ & & 0 & 0 \\
\hline & \multicolumn{3}{|l|}{ Indetermination } & 0 & 0 \\
\hline & \multicolumn{3}{|c|}{ Genericization } & 0 & 0 \\
\hline & \multicolumn{3}{|c|}{ Specification (Individualization) } & 0 & 0 \\
\hline \multirow[t]{2}{*}{ Impersonalization } & \multicolumn{3}{|c|}{ Abstraction } & 0 & 0 \\
\hline & \multicolumn{3}{|l|}{ Objectivation } & 0 & 0 \\
\hline
\end{tabular}

\section{Conclusion}

Dans la perspective du CDA, la publication de Jokowi et Prabowo sur bali.gazette et LesEchos.fr ne sont pas dans le temps vide, mais cette publication est étroitement liée à une politique-économique dans des séries d'événements politiques en Indonésie dont le pic sera l'élection présidentielle de 2019. A travers le cadre du van Leeuwen, à savoir trois types de transformation discursive : la suppression, l'allocation de rôles, et la substitution, nous pourrions comprendre les représentations transmisses par les deux journalistes de deux médias en ligne : bali-gazette.com et LesEchos.fr. Ils ont représenté Jokowi Widodo positivement que Prabowo Subianto. Dans le perspectif sémiologique social van Leeuwen, la représentation égale des deux journalistes pourraient être interprétée qu'ils favorisent ou soutiennent Jokowi de regagner à l'élection présidentielle en 2019. Ce soutien repose sur un motif ou une combinaison de deux ou trois motifs, à savoir le motif idéologique, politique et économique. Mais dans la perspective du positivisme, il semble que les publications des deux candidats à la présidentielle ci-dessus soient objectives car elles sont conformées aux faits de la communauté et aux statistiques. Cependant, du point de vue du CDA, la subjectivité des journalistes est très évidente dans le choix des trois types de transformation discursive ci-dessus.

\section{Références}

Fairclough, N. (1995). Critical Discourse Analysis: The Critical Study of Language. London: Longman. https://doi.org/10.2307/329335

Halliday, M. A. K. (1985). An Introduction to Functional Grammar. London: Edward Arnold. https://doi.org/10.2307/415759 
Kress, G., \& van Leeuwen, T. (1996). Multimodal Discourse: The Modes and Media of Contemporary

Communication. London: Bloomsbury Academic.

Potter, J. (2005). Representing Reality-Discourse, Rhetoric \& Social Construction. London: SAGE.

Schneider, F. (2013). Getting the Hang of Discourse Theory. Retrieved from

http://www.politicseastasia.com/studying/getting-the-hang-of-discourse-theory/

Sperber, D. (1996). Explaining Culture - A Naturalistic Approach. Oxford: Blackwell Publishing.

Sudaryanto. (1993). Metode dan Aneka Teknik Analisis Bahasa. Yogyakarta: Duta Wacana University Press.

Sujiman., P., \& van Zoest, A. (1992). Serba-Serbi Semiotika. Jakarta: Gramedia.

Tebba, S. (2005). Jurnalistik Baru. Jakarta: Kalam Indonesia.

van Leeuwen, T. (2008). Discourse and Practice-New Tools for Critical Discourse Analysis. Oxford: Oxford

University Press.

van Leeuwen, T. (2005). Introducing Social Semiotics. New York: Routledge. 